\section{Rhabdomyolysis after group C streptococcal infection}

\author{
Hilde Haugedal Nordal, \\ Bård Reiakvam Kittang, ${ }^{2}$ \\ Laurence A. Bindoff ${ }^{3,4}$
}

'Department of Medicine/Rheumatology, Haukeland University Hospital, Bergen, Norway; ${ }^{2}$ Institute of Medicine, University of Bergen, Norway; ${ }^{3}$ Institute of Clinical Medicine, University of Bergen, Norway; ${ }^{4}$ Department of Neurology, Haukeland University Hospital, Bergen, Norway

\section{Abstract}

We describe a young woman with a group $\mathrm{C}$ streptococcal throat infection complicated by rhabdomyolysis. Muscle biopsy from quadriceps was normal, and molecular studies showed no evidence of direct microbial invasion. This is only the second case in which the usually benign group $\mathrm{C}$ streptococcus has been linked with muscle destruction.

\section{Introduction}

Acute rhabdomyolysis during bacterial infection is recognised, but rare. ${ }^{1}$ Muscle destruction can arise either due to direct invasion or secondary to toxic damage. Group C streptococci (GCS) are usually associated with minor pharyngeal or dermatological infections and only one report has previously documented rhabdomyolysis in a patient with GCS infection. ${ }^{2}$

\section{Case Report}

A previously healthy, 21-year old woman was admitted to our hospital with a three day history of pain in the back and lower extremities. Nine days before admission she had had a sore throat and cough that had disappeared without treatment after approximately four days. Two days later she developed pain in the upper extremities, but not in the muscles of the jaw, eye or throat. On the day of admission she developed swelling of the right leg and was referred to us with a tentative diagnosis of deep vein thrombosis. Apart from small doses of paracetamol and ibuprofen for pain relief, her only regular medication was a low dose combined oral contraceptive (etinylestradiol/ levogestrel). She had not taken any herbal medications. There was no history of direct trauma, but she was an active player of floor- ball. Examination on admission showed a low grade fever of $38.1^{\circ} \mathrm{C}$ and bilaterally enlarged tonsils. The right calf was approximately $1 \mathrm{~cm}$ larger in diameter than the left. She had pain in the muscles of both legs on palpation and Homan's sign was positive. There were no signs of inflammation involving the skin or subcutaneous tissues of the calf and the remainder of the examination was normal. Two days after admission she also developed a general swelling of the left hand.

Initial blood tests (with normal range values in parentheses) were as follows: C-reactive protein $55 \mathrm{mg} / \mathrm{L}$ ( CRP; <5), d-dimer $7.03 \mathrm{mg} / \mathrm{L}$ (0.00-0.50), s-creatine kinase $35506 \mathrm{U} / \mathrm{L}$ (35$210)$, s-myoglobin $2848 \mu \mathrm{g} / \mathrm{L}(<50)$, erythrocyte sedimentation rate $36 \mathrm{~mm},(\mathrm{ESR} ;<20)$ leukocyte count $12.9 \times 10^{9} / \mathrm{L}(3.5-11.0)$, neutrophil count $8.9 \times 10^{9} / \mathrm{L}$ (1.7-8.2), lymphocyte count 2.5 $\times 10^{9} / \mathrm{L}(0.7-5.3)$, monocyte count $1.02 \times 10^{9} / \mathrm{L}$ (0.04-1.3), eosinophil count $0.4 \times 10^{9} / \mathrm{L}(0.0$ $0.7)$, basophil count $0.0 \times 10^{9} / \mathrm{L} \quad(0.0-0.3)$, platelet count $445 \times 10^{\circ} / \mathrm{L}(165-387)$, s-creatinine $51 \mu \mathrm{mol} / \mathrm{L}$ (45-90), s-potassium 4.4 $\mathrm{mmol} / \mathrm{L} \quad(3.5-5.0)$ and $\mathrm{s}$-phosphorus 0.99 $\mathrm{mmol} / \mathrm{L}(0.85-1.50)$. U-stix was positive for hemoglobin $3+$ and protein $1+$. Urinemicroscopy showed physiological urine. Blood cultures were not performed as she had no signs of bacteremia/sepsis. Ultrasound examination showed no sign of deep vein thrombosis and due to the clear indication of muscular pathology, venography was not performed. No further imaging was performed.

Viral myositis was suspected, but antibodies against enterovirus, parainfluenza virus, adenovirus, influenzavirus A and B, herpes simplex virus, varicella zoster virus, measles virus, parvovirus, Epstein Barr virus and cytomegalovirus were negative or only showed evidence of previous infection. Group C streptococci (GCS) were cultured from a tonsillar swab and antistreptolysin (ASO) and anti DNAse B-titers showed a typical rise and fall indicating active infection. ASO titre was initially 573 (normal range $<400 \mathrm{IU} / \mathrm{mL}$ ) rising to 2490 two weeks later and falling to $1720 \mathrm{IU} / \mathrm{mL}$ after 6 weeks; anti DNAse B titers were 250-393-360 IU/mL (normal range $<200 \mathrm{IU} / \mathrm{mL}$ ) at the same time points.

Her rhabdomyolysis was treated with forced alkaline diuresis, but on the fourth day she developed a skin rash when given sodium hydrogencarbonate and received only fluids and a diuretic thereafter. The CK level fell slowly. On day 3 after admission, when positive serology for streptococci was identified and the tonsillar swab showed growth of group C streptococci, she started a 14 days course of oral penicillin. The pain and stiffness in her muscles gradually resolved as did the swelling of the right leg and left hand.

Muscle biopsy was performed under local anaesthesia on day 5 after admission. Muscle
Correspondence: Hilde Haugedal Nordal, Department of Rheumatology, Haukeland University Hospital 5021 Bergen, Norway.

E-mail: hilde.haugedal.nordal@helse-bergen.no

Key words: infections, muscle disorders, rhabdomyolysis, group C streptococcus.

Contributions: HHN treated the patient when she was hospitalized, found references, participated in the discussion and wrote the case report; BRK found references, planned the sequencing of the bacterial 16rRNA in the muscle and participated in the discussion from the view of an infectious disease specialist; LAB evaluated the patient neurologically, did the muscle biopsy, saw the patient on follow up, initiated the work with the article and participated in the discussion from the view of a specialist in muscle disease.

Acknowledgements: we are grateful to Professor Sigurd Lindal, Department of Pathology, University Hospital of Troms $ø$, Norway and Rebecca I. Breistein, Department of Microbiology, Haukeland University Hospital, for performing the diagnostic investigation of muscle.

Conflict of interest: the authors report no conflicts of interest.

Received for publication: 1 July 2010.

Revision received: 24 September 2010.

Accepted for publication: 27 September 2010.

This work is licensed under a Creative Commons Attribution 3.0 License (by-nc 3.0).

(C) Copyright H.H. Nordal et al., 2010

Licensee PAGEPress, Italy

Infectious Disease Reports 2010; 2:e15

doi:10.4081/idr.2010.e15

from the right quadriceps was examined by light- and electron microscopy and showed no evidence of necrosis, no inflammatory cell infiltrate, no accumulation of lipid or glycogen and no evidence of MHC1 upregulation. Enzyme histochemical staining showed normal myophosphorylase, myoadenylate deaminase, phosphofructokinase, aldolase, lactate dehydrogenase, cytochrome oxidase and succinate dehydrogenase activities. Electron microscopy showed a normal muscle ultrastructure. Subsequently, we have examined her muscle for the presence of bacteria. We found no evidence of GCS in her muscle on bacterial culture, and bacterial DNA was not amplified from the muscle biopsy using a broad-range PCR targeting the $16 \mathrm{~S}$ ribosomal RNA gene as described, ${ }^{3}$ with the following primers: F: 5'- TTG-GAG-AGT-TTG-ATC-MTG-C$3^{34}$ and R: 5'-GTA-TTA-CCG-CGG-CTG-CTG-3' ${ }^{3,6}$

She was discharged after 17 days in hospital, and returned to work after 4 weeks after discharge. One month later, s-CK and s-CRP were normal and $s$-CK has remained normal (5 
months follow up). She has returned to work and playing floorball and has no further muscle related symptoms.

\section{Discussion}

We describe a patient with rhabdomyolysis complicating a streptococcal throat infection. The common causes of rhabdomyolysis, including both metabolic and structural myopathies, were excluded by muscle biopsy. Initially, we suspected a viral aetiology, but serological tests for the common viral causes were negative. The presence of a throat infection with GCS cultured from a throat swab and rising anti-streptolysin and anti-DNAse $B$ titres raised the possibility that this might be the cause of her rhabdomyolysis.

GCS are $\beta$-haemolytic streptococci that occasionally cause pharyngitis or mild skin infections and are only rarely associated with severe, invasive infections. There have, however, been reports of GCS associated with disease manifestations including primary bacteremia, arthritis, endocarditis, meningitis, pneumonia, necrotising fasciitis, myositis and even fatal toxic shock-like syndrome has been described..$^{7.9}$ Acute rhabdomyolysis during bacterial infection is recognised, but rare. The most commonly implicated bacterial pathogen is Legionella, although streptococci, staphylococci and gram-negative bacteria have also been reported in association with muscle destruction. ${ }^{1}$ A search of The Cochrane Library, PubMed and Medline with the keywords "group C streptococci" and "rhabdomyolysis" revealed only one case report describing a severe invasive GCS-infection associated with rhabdomyolysis and disseminated intravascular coagulation in a previously healthy adult. ${ }^{2}$

While there appears to be a relationship between a mild GCS throat infection and rhabdomyolysis, the nature of any causal association, particularly the pathophysiological mechanism linking them, remains unclear. Our patient had no history of recent muscle injury, and, while the biopsy was taken 2 days after starting oral penicillin, we could not isolate streptococcal DNA from her muscle. She did not develop symptoms or clinical signs compatible with streptococcal toxic shock syndrome or necrotising streptococcal myositis. The clinical picture could reflect the first stages of pyomyositis that is characterised by local swelling, relatively mild pain and fever, ${ }^{10}$ but pyomyositis is most often associated with recent muscle trauma, bacteremia and normal CK values. Furthermore, the lack of inflammatory cell infiltrate and absence of bacterial DNA in the muscle biopsy indicate that the marked CK elevation cannot be explained by general bacterial muscle invasion. Moreover, the muscle biopsy was taken to rule out underlying metabolic disorders from a muscle without obvious swelling. Endotoxins or exotoxins are believed to play a role in the pathogenesis of Legionella-associated rhabdomyolysis, ${ }^{11,12}$ and the cysteine protease speB was shown to enhance local skin, subcutaneous tissue and muscle damage of strains of Streptococcus pyogenes in a mouse model. ${ }^{13}$ However, the GCS isolate associated with a previous case of severe systemic infection and rhabdomyolysis did not show sign of exotoxin activity inducing T-cell mitogenicity. ${ }^{2}$ Unfortunately, our GCS isolate was not available for further analysis. Hence, we were neither able to check for the presence of bacterial endotoxins nor for a possible mitogenic response.

This is only the second case in which GCS infection has been linked with rhabdomyolysis. In contrast to the previous case, our patient had a relatively mild GCS-infection, and we found no obvious clinical, microbiological, molecular or histological signs of muscle invasion. While the exact mechanism remains unknown, we feel it is important to alert other physicians to the possibility that GCS can be associated with significant muscle destruction.

\section{References}

1. Singh U, Scheld WM. Infectious etiologies of rhabdomyolysis: three case reports and review. Clin Infect Dis 1996;22:642-9.

2. Ojukwu IC, Newton DW, Luque AE, et al. Invasive Group C Streptococcus infection associated with rhabdomyolysis and disseminated intravascular coagulation in a previously healthy adult. Scand J Infect Dis 2001;33:227-9.

3. Kommedal 0, Kvello K, Skjastad R, et al.
Direct 16S rRNA gene sequencing from clinical specimens, with special focus on polybacterial samples and interpretation of mixed DNA chromatograms. J Clin Microbiol 2009;47:3562-8.

4. Petti CA, Bosshard PP, Brandt ME, et al. Interpretive criteria for identification of bacteria and fungi by DNA target sequencing. Approved guideline. 2008; Document MM18-A, vol. 28, no 12. Clinical and Laboratory Standard Institute, Wayne, PA.

5. Bosshard PP, Zbinden R, Altwegg M. Turicibacter sanguinis gen. nov., sp. nov., a novel anaerobic, Gram-positive bacterium. Int J Syst Evol Microbiol 2002;52:1263-6.

6. Edwards U, Rogall T, Blocker $\mathrm{H}$, et al. Isolation and direct complete nucleotide determination of entire genes. Characterization of a gene coding for $16 \mathrm{~S}$ ribosomal RNA. Nucleic Acids Res 1989;17: 7843-53.

7. Broyles LN, Van Beneden C, Beall B, et al. Population-based study of invasive disease due to $\beta$-hemolytic streptococci of groups other than A and B. Clin Infect Dis 2009; 48:706-12.

8. Ekelund K, Skinhoj P, Madsen J, Konradsen HB. Invasive group A, B, C and G streptococcal infections in Denmark 1999-2002: epidemiological and clinical aspects. Clin Microbiol Infect 2005;11:56976.

9. Korman TM, Boers A, Gooding TM. et al. Fatal case of toxic shock-like syndrome due to group $\mathrm{C}$ streptococcus associated with superantigen exotoxin. J Clin Microbiol 2004;4:2866-9.

10. Crum-Cianflone NF. Bacterial, fungal, parasitic, and viral myositis. Clin Microbiol Rev 2008;21:473-94.

11. Shah A, Check F, Baskin S, et al. Legionnaires' disease and acute renal failure: case report and review. Clin Infect Dis 1992;14:204-7.

12. Wong KH, Moss CW, Hochstein DH, et al. "Endotoxicity" of the Legionnaires' disease bacterium. Ann Intern Med 1979;90: 624-7.

13. Saouda M, Wu W, Conran P, Boyle MD. Streptococcal pyrogenic exotoxin B enhances tissue damage initiated by other Streptococcus pyogenes products. J Infect Dis 2001;184:723-31. 